\title{
Cloxacillin-resistant staphylococci in a children's hospital
}

\author{
M. B. SKIRROW'1 AND K. B. ROGERS \\ From the Department of Microbiology, Children's Hospital, Birmingham
}

SYNOPSIS Two hundred and thirty-seven selected, penicillin-resistant isolates of Staph. aureus collected over a 13-month period were examined retrospectively for cloxacillin/methicillin resistance; 44 were found to be resistant. Six other strains which had been preserved from an earlier period were also found to be resistant. All 50 cloxacillin-resistant strains were in addition resistant to streptomycin and several other antibiotics. According to their resistance patterns and reactions to phages, they could be classified into two categories.

The proportion of cloxacillin-resistant strains among the total number of staphylococci isolated during the 13 -month period was estimated to be $2 \cdot 7 \%$. Children most at risk from infection were those undergoing long-term hospital treatment and on several occasions children transferred from other hospitals in the region were found to be carrying resistant strains. Most infections caused by these strains were minor but they were nevertheless capable of producing severe illness and they were responsible for $33 \%$ of all staphylococcal wound infections.

Strains of Staphylococcus aureus resistant to methicillin, and therefore cloxacillin, remained rare for several years after their first isolation by Jevons in 1960 (Jevons, 1961). By 1964 they constituted 0.97\% of staphylococci, mostly of hospital origin, sent to the Central Public Health Laboratory, Colindale, for phage typing (Dyke, Jevons, and Parker, 1966). Since then they have been found with increasing frequency, particularly in Europe where they have caused serious infections (Benner and Kayser, 1968).

With rare exceptions cloxacillin/methicillin-resistant strains of Staph. aureus consist of mixed populations of cells in which only a small minority show a high degree of resistance to these antibiotics; most of the cells are only slightly less sensitive than those of fully sensitive strains. This heterogenous resistance is likely to be overlooked if reliance is placed solely on routine disc-diffusion methods of sensitivity testing, and for this reason cloxacillin-resistant staphylococci are probably more widespread in Britain than the few reports from hospital laboratories suggest (Colley, McNicol, and Bracken, 1965; Turner and Cox, 1967; Churcher, 1968). This is illustrated by our experiences in this laboratory, where cloxacillin-resistant staphylococci passed un-

${ }^{1}$ Present address: Public Health Laboratory, Royal Infirmary, Castle Street, Worcester.

Received for publication 25 June 1970. recognized until special techniques for their detection were introduced. At that tıme, in October 1968, we used such methods to re-examine a collection of Staph. aureus cultures which had been preserved during the preceding year. This paper is an account of this study and of the cloxacillin-resistant staphylococci that were found.

\section{Methods}

SOURCE OF STAPHYLOCOCCI

Coagulase-positive staphylococci isolated from routine material were selected for preservation on 0 the following grounds: possession of multiple antibiotic resistance; association with wound infections or cross infection; association with severe infections such as osteomyelitis and pneumonia. Two hundred $\%$ and seventy-two staphylococci had been preserved $N$ in this way between September 1967 and October N 1968. Of these, 35 were penicillin-sensitive and as a result they were excluded from the study. The remaining 237 penicillin-resistant isolates were tested $\stackrel{0}{\varnothing}$ for cloxacillin resistance by the methods detailed $\stackrel{\Phi}{\mathcal{D}}$ below. Six additional isolates which had been preserved at the beginning of 1967 on account of resistance to a wide range of antibiotics were also tested. The majority of isolations were made from children $\vec{\Phi}$ on the wards of the hospital or attending the out- 
patient and casualty departments; some were obtained from members of the hospital staff.

DETERMINATION OF ANTIBIOTIC SENSITIVITIES At the time of their original isolation, all coagulasepositive staphylococci were tested against a panel of antibiotics by the disc-diffusion method on a blood agar medium to which had been added $5 \%$ lysed horse blood (Harper and Cawston, 1945). Antibiotics were applied in the form of an Oxoid Multodisc $616 \mathrm{E}$ containing penicillin $\mathrm{G}$ (1.5 units), streptomycin $(10 \mu \mathrm{g})$, chloramphenicol (10 $\mu \mathrm{g})$, tetracycline $(10 \mu \mathrm{g})$, erythromycin (10 $\mu \mathrm{g})$, sulphadimidine $(100 \mu \mathrm{g})$, neomycin $(30 \mu \mathrm{g})$, cloxacillin $(5 \mu \mathrm{g})$. Lincomycin $(2 \mu \mathrm{g})$ and cepholoridine $(25 \mu \mathrm{g})$ were added as separate discs. Plates were incubated overnight at $37^{\circ} \mathrm{C}$.

Staphylococci subsequently found to be cloxacillin-resistant were further tested against fusidic acid $(10 \mu \mathrm{g})$, novobiocin $(5 \mu \mathrm{g})$, gentamicin $(2 \mu \mathrm{g})$, vancomycin $(25 \mu \mathrm{g})$, rifamycin $(10 \mu \mathrm{g})$, trimethoprim (2.5 $\mu \mathrm{g})$, and sulphamethoxazole $(50 \mu \mathrm{g})$; the last two were tested both singly and in combination in order to detect synergy. These additional tests were done on Oxoid diagnostic sensitivity test (DST) medium containing $0.25 \%$ lysed horse blood (Darrell, Garrod, and Waterworth, 1968). Plates were inoculated with six-hour broth cultures diluted to give semi-confluent growth and incubated overnight at $37^{\circ} \mathrm{C}$ in air.

\section{DETECTION OF CLOXACILLIN RESISTANCE Cloxacillin agar test (Turner and Cox, 1967)}

Nutrient agar plates containing $1.6 \mu \mathrm{g}$ and $100 \mu \mathrm{g}$ cloxacillin were inoculated with a drop of undiluted overnight broth culture of each organism. Plates were incubated at $37^{\circ} \mathrm{C}$ and examined after 24 and 48 hours for the presence of growth.

\section{Methicillin disc-diffusion test using $5 \% \mathrm{NaCl}$ agar (Churcher, 1968)}

Nutrient agar plates containing $5 \%$ sodium chloride were inoculated with streaks of four-hour broth cultures of the test organisms. Paper discs containing $10 \mu \mathrm{g}$ methicillin were applied to the inoculated areas and the plates incubated at $37^{\circ}$ overnight.

\section{Phage typing}

All cloxacillin-resistant staphylococci were phage typed at the Cross Infection Reference Laboratory, Colindale, London.

\section{Results}

TESTS FOR CLOXACILLIN RESISTANCE Of the 237 penicillin-resistant staphylococci tested,
183 failed to grow on cloxacillin agar at either concentration and were inhibited to within 6 to $10 \mathrm{~mm}$ from the edge of a $10-\mu \mathrm{g}$ disc of methicillin on $5 \%$ salt agar; these organisms were regarded as fully sensitive.

Forty-four isolates grew on both concentrations of cloxacillin agar and grew up to or within $1 \mathrm{~mm}$ of a methicillin disc; these were regarded as resistant.

Ten isolates grew on plates containing $1.6 \mu \mathrm{g}$ cloxacillin but not on those containing $100 \mu \mathrm{g}$; these were inhibited to within 4 to $9 \mathrm{~mm}$ of a methicillin disc and were not considered to be truly resistant. They conform to the 'borderline' strains of Turner and Cox (1967); all showed multiple antibiotic resistance and all were isolated from children who had received long-term antibiotic therapy (nine with cystic fibrosis and one with a chronically infected sinus associated with a cardiac pacemaker).

All six multiple-resistant isolates obtained earlier in 1967 were found to be cloxacillin-resistant by the above methods, thus bringing the total number of resistant strains in this series to 50 .

Growth on cloxacillin agar was in every case confined to a few colonies, representing about one bacterium in ten million of the original inoculum. This is characteristic of the heterogenous type of resistance exhibited by most cloxacillin-resistant strains of Staph. aureus. No isolate resembled the strain described by Stewart and Holt (1963) in which most of the bacterial population were resistant to high concentrations of methicillin.

The methicillin disc test was found to be the more convenient of the two tests, mainly because results could be obtained more quickly. However, in the light of Annear's recent discovery that resistance is more easily demonstrated at low-temperature incubation, it appears that the most reliable screening test for the detection of these strains is by disc-diffusion using $10 \mu$ methicillin on plain nutrient agar

\begin{tabular}{lll}
\hline Phage Pattern & No. of Strains & Phage Category \\
\hline $29 / 77$ & 15 & \\
$29 / 77 / 84$ & 9 & \\
$29 / 79 / 77 / 84$ & 8 & \\
$29 / 79 / 77$ & 3 & \\
$29 / 75 / 77$ & 2 & \\
$29 / 75 / 77 / 84$ & 1 & \\
$29 / 79 / 75 / 77 / 84$ & 1 & \\
$29 / 79 / 77 / 85$ & 1 & \\
$29 / 79 / 77 / 83 \mathrm{~A} / 84 / 85$ & 1 & \\
Total & 41 & \\
$53 / 85$ plus incomplete or & & - \\
complete reactions with & 7 & \\
29 and 42D & 2 & \\
Not typeable & & \\
\hline
\end{tabular}

Table I Phage patterns of cloxacillin-resistant staphylococci 


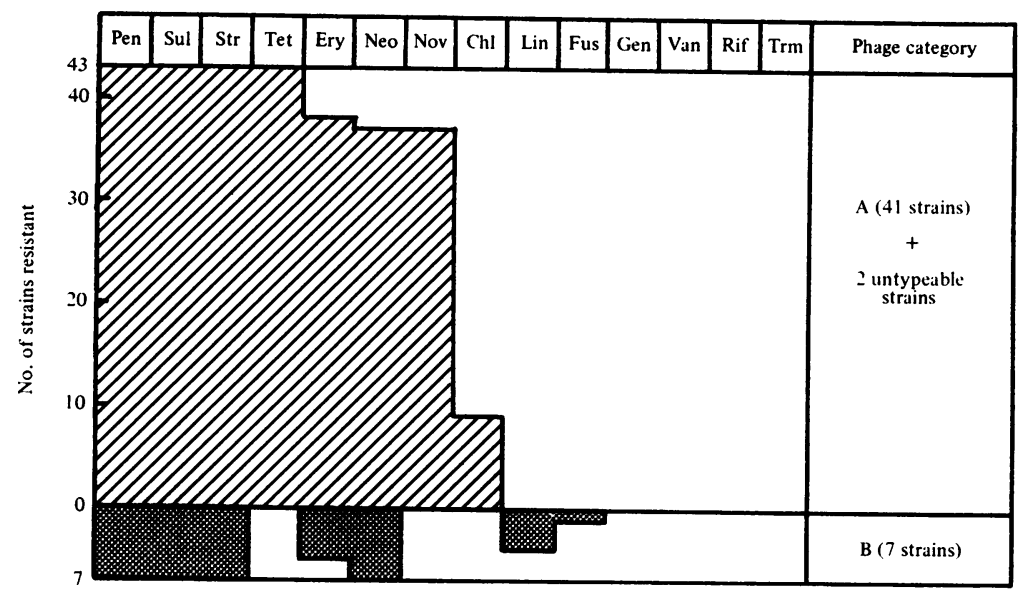

Fig. Antibiotic sensitivities of cloxacillin-resistant staphylococci. Pen = penicillin $G$; Sul= sulphadimidine; Str = streptomycin; Tet = tetracycline; Ery = erythromycin; $\mathrm{NeO}=$ neomycin; Nov = novobiocin; Chl = chloramphenicol; Lin $=$ lincomycin; Fus = fusidic acid; Gen = gentamicin; Van = vancomycin; Rif = rifamide; Trm =trimethoprim.

incubated at $30^{\circ} \mathrm{C}$ instead of $37^{\circ} \mathrm{C}$ (Hewitt, Coe, and Parker, 1969).

\section{PROPERTIES OF CLOXACILLIN-RESISTANT STAPHYLOCOCCI \\ Phage typing}

Apart from two isolates which were untypable, these staphylococci could be divided into two categories. Category A contained 41 isolates which were lysed by phages 29 and 77; 19 were also lysed by phage 84 and 14 by phage 79 (Table I). Category B contained seven isolates which were lysed by group III phages 53/85 and all showed at least partial lysis to phages 29 and 42D; the differences between these patterns of lysis were not significant and isolates in this category were therefore indistinguishable on these grounds.

Sensitivity to chemotherapeutic agents and antibiotics other than cloxacillin

All cloxacillin-resistant isolates possessed multiple antibiotic resistance. The Figure portrays diagrammatically the resistance patterns of these staphylococci which were by definition resistant to penicillin
G. In addition, all were resistant to sulphadi- $\frac{\mathbb{O}}{\mathrm{O}}$ midine and streptomycin. Resistance to other anti- $\frac{1}{\mathrm{~N}}$ biotics differed according to the phage category: all 2 those in category $\mathbf{A}$ and the two untypable isolates $\vec{\varphi}$ were resistant to tetracycline and most also to $\rightarrow$ erythromycin, neomycin (kanamycin), and novobiocin. On the other hand, category $B$ isolates were relatively sensitive to tetracycline and novobiocin; three of these were resistant to lincomycin and one to fusidic acid. All 50 isolates (both categories) $\stackrel{\circ}{\otimes}$ were sensitive to gentamicin, vancomycin, rifamide, $\stackrel{\varrho}{\rightarrow}$ and trimethoprim. Synergy between trimethoprim and sulphamethoxazole was clearly demonstrated in all cases, although there was no inhibition of growth to $50 \mu \mathrm{g}$ of sulphamethoxazole alone.

\section{Pathogenicity}

The 50 cloxacillin-resistant staphylococci in this series were isolated from 47 persons. Twelve children $\delta$ and one nurse were found to be harbouring them without apparent ill effect. In 10 instances the 을 staphylococci were recovered from the upper respir-? atory tract and in three from the perineum or vulva. Twenty-seven isolates were found in association with

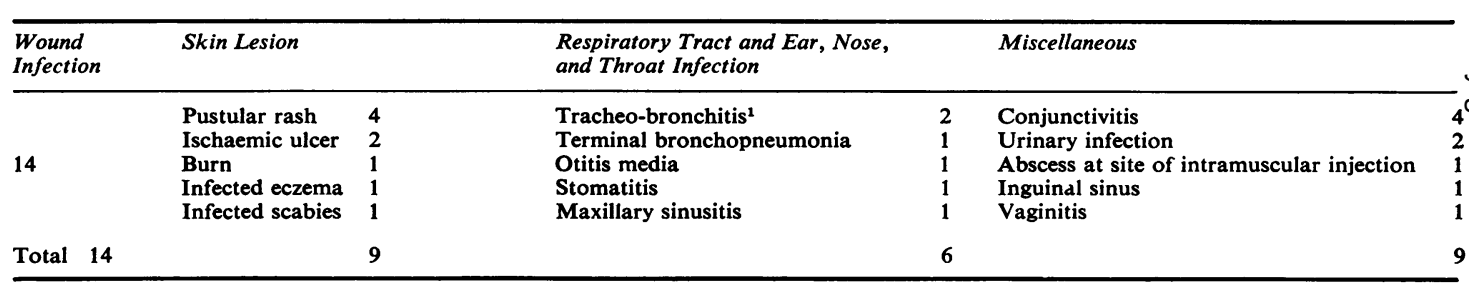

Table II Lesions associated with cloxacillin-resistant staphylococci

${ }^{1}$ Tracheal aspiration during intensive care. 
minor infections, often with other bacteria. Seven were the cause of more severe types of infection, but in no case was death directly attributable to their presence. The most serious infection was in a $2 \frac{1}{2}$ year-old boy who developed a staphylococcal empyema after pneumonectomy.

The types of lesion associated with these organisms are shown in Table II. Infections of surgical wounds form the largest group (14) with infections of the skin forming the second largest (9); six of the latter were associated with staphylococci belonging to phage category B. Factors which predisposed to infection were present in many of the infected children. Almost two-thirds of them were less than 18 months old and many had chronic debilitating diseases which had required prolonged treatment in hospital. However, of 22 children with cystic fibrosis who had staphylococci in the sputa, only two (siblings) were carrying a cloxacillin-resistant strain.

\section{INCIDENCE OF CLOXACILLIN-RESISTANT}

STAPHYLOCOCCI

During the period covered by this study a total of 1,649 coagulase-positive staphylococci were made in this laboratory. As already described, 272 of these had been preserved and tested for cloxacillin resistance and they included all those showing multiple antibiotic resistance. Since in this study and in that of Turner and Cox (1967) all cloxacillin-resistant staphylococci were found to possess multiple antibiotic resistance, it is reasonable to assume that all such organisms among the 1,649 had been detected. On this assumption the overall proportion of cloxacillin-resistant isolates was $2.7 \%$. This is a crude figure which does not take into account the possibility that the same organism may have been isolated from more than one patient.

SOURCES OF INFECTION WITH CLOXACILLINRESISTANT STAPHYLOCOCCI

In all cases but one, infected children had been or were in hospital when the staphylococci were found; the exception was an Asian boy who had a chronically infected sinus of the groin. There were several instances in which children appeared to have brought resistant strains into the Children's Hospital when transferred from other hospitals in the Midlands. Direct case-to-case transmission of a strain was seldom observed, but cloxacillin-resistant organisms were responsible for $33 \%$ of all staphylococcal wound infections. On no occasion were cloxacillinresistant staphylococci found to be causing sepsis in members of the hospital staff; moreover, in an investigation of an instance of suspected cross infection we failed to recover an organism of this type from 42 of the staff and 17 children who were contacts of a heavily colonized little girl.

\section{EFFECT OF TREATMENT WITH CLOXACILLIN} When these staphylococci were first isolated, resistance to cloxacillin was not suspected and some of the children were treated for their infections with this antibiotic. Of eight patients so treated, the staphylococci persisted in six: two had infected wounds, one had an infected plaster sore, and three (two with cystic fibrosis) continued to carry a small number of staphylococci in the respiratory tract. A 7-month-old baby's wound became infected whilst receiving cloxacillin (62 $\mathrm{mg}$ six hourly by mouth) as a prophylactic against staphylococcal infection.

\section{USE OF PENICILLINS IN THE HOSPITAL}

Resistance to cloxacillin among strains of Staph. aureus is due to a tolerance which extends to all penicillins. Hence, the selection and survival of such strains is probably influenced by the extent to which penicillins are used. Table III shows the quantities of penicillins of all types dispensed by the Birmingham Children's Hospital during the past three years.

\begin{tabular}{lccc}
\hline & 1966 & 1967 & 1968 \\
\hline Cloxacillin & 5 & 6 & 5 \\
All other penicillins & 25 & 37 & 35 \\
Total & 30 & 43 & 40 \\
\hline
\end{tabular}

Table III Quantities of penicillins $(\mathrm{kg})$ dispensed at the Birmingham Children's Hospital 1966-68

\section{Discussion}

Owing to the existence of specialized and intensive care units at this hospital, children are commonly referred from other hospitals in the region. Such children have often suffered long periods in hospital and when admitted are usually found to be carrying resistant strains of bacteria. It is not surprising, therefore, that cloxacillin-resistant staphylococci have been found in this hospital. The overall incidence of $2.7 \%$ is close to the $3.2 \%$ quoted by Churcher (1968) from Plymouth General Hospital; figures given by other authors are not comparable because they are based on collections of staphylococci selected by different criteria.

Strains lysed by both phage 29 and group III phages, ie, like our category $A$ isolates, have been seen in a number of hospitals in Britain in recent years (M. T. Parker, personal communication); these appear to differ from the strains reported by 
Colley et al (1963), Turner and Cox (1967), and Churcher (1968). Strains lysed solely by phage 29 but with inhibition reactions with group III phages have been recorded from several countries in Europe.

The types of infection associated with these staphylococci follow the pattern of those previously reported. Probably all the infections were acquired in hospital and children residing in hospital for long periods and suffering from chronic debilitating diseases were especially prone to infection. On the whole the infections were of minor severity, but there can be no doubt that on occasion these strains can cause serious infections which threaten life, as exemplified by the little boy who developed an empyema following pneumonectomy.

The choice of antibiotics suitable for the treatment of such infections is restricted. Cloxacillin/methicillin-resistant strains are also resistant to the cephalosporins (Hewitt and Parker, 1968). However, in our series almost all the resistant isolates were sensitive to lincomycin and fusidic acid and these are relatively non-toxic, powerful antibiotics which can be taken by mouth. In addition, all strains were sensitive to gentamicin, rifamycin, trimethoprim/sulphamethoxazole, and vancomycin.

We are indebted to Dr M. T. Parker and $\mathrm{Mr} \mathrm{J.} \mathrm{H.}$
Hewitt of the Cross Infection Reference Laboratory, Colindale, London, for phage typing the 50 strains of staphylococci and for their helpful comments.

\section{References}

Benner, E. J., and Kayser, F. H. (1968). Growing clinical significance of methicillin-resistant Staphylococcus aureus. Lancet, 2, 741744.

Churcher, G. M. (1968). A screening test for the detection of methicillin-resistant staphylococci. J. clin. Path., 21, 213-217.

Colley, E. W., McNicol, M. W., and Bracken, P. M. (1965). Methicillin-resistant staphylococci in a general hospital. Lancet, 1 , 595-597.

Darrell, J. H., Garrod, L. P., and Waterworth, P. M. (1968). Trimethoprim: laboratory and clinical studies. J. clin. Path., 21, 202-209.

Dyke, K. G. H., Jevons, M. P., and Parker, M. T. (1966). Penicillinase production and intrinsic resistance to penicillins in Staphylococcus aureus. Lancet, 1, 835-838.

Harper, G. J., and Cawston, W. C. (1945). The in-vitro determination of the sulphonamide sensitivity of bacteria. J. Path. Bact., 57 , 59-66.

Hewitt, J. H., Coe, A. W., and Parker, M. T. (1969). The detection of methicillin resistance in Staphylococcus aureus. J. med. Microbiol. , 2, 443-456.

Hewitt, J. H., and Parker, M. T. (1968). Sensitivity of penicillinaseforming strains of Staphylococcus aureus and of their penicillinase-negative variants to cephaloridine, cephalothin, methicillin, and benzylpenicillin. J. clin. Path., 21, 75-84.

Jevons, M. P. (1961). 'Celbenin'-resistant staphylococci. (Letter to Editor.) Brit. med.J., 1, 124-125.

Stewart, G. T., and Holt, R. J. (1963). Evolution of natural resistance to the newer penicillins. Brit. med.J., 1, 308-311.

Turner, G. C., and Cox, P. E. (1967). Resistance to cloxacillin among hospital staphylococci. J. clin. Path., 20, 870-874. 